\title{
Inflammation and Fatigue Dimensions in Advanced Cancer Patients and Cancer Survivors
}

\author{
An Explorative Study \\ Pleun J. de Raaf, MD; Stefan Sleijfer, MD, PhD; Cor H.J. Lamers, PhD²; Agnes Jager, MD, PhD'; \\ Jan Willem Gratama, $\mathrm{PhD}^{2}$; and Carin C. D. van der Rijt, MD, $\mathrm{PhD}^{1}$
}

\begin{abstract}
BACKGROUND: Inflammation may underlie cancer-related fatigue; however, there are no studies that assess the relation between fatigue and cytokines in patients with advanced disease versus patients without disease activity. Furthermore, the relation between cytokines and the separate dimensions of fatigue is unknown. Here, association of plasma levels of inflammatory markers with physical fatigue and mental fatigue was explored in advanced cancer patients and cancer survivors. METHODS: A total of 45 advanced cancer patients and 47 cancer survivors completed the subscales Physical Fatigue and Mental Fatigue of the Multidimensional Fatigue Inventory. Plasma concentrations of C-reactive protein (CRP), interleukin-1 receptor antagonist (IL-1-ra), interleukin-6 (IL-6), interleukin-8 (IL-8), and neopterin were measured. Nonparametric tests were used to assess differences in fatigue intensity and levels of inflammatory markers and to determine correlation coefficients between the fatigue dimensions and inflammatory markers. RESULTS: Compared with cancer survivors, patients with advanced cancer had higher levels of physical fatigue (median 16 vs $9, P<$ .001) and mental fatigue (median $11 \mathrm{vs} 6, P=.01$ ). They also had higher levels of all cytokines $(P<.01)$. In advanced cancer, CRP $(r=$ $0.49, P=.001)$, IL-6 $(r=0.43, P=.003)$, IL-1-ra $(r=0.32, P=.03)$, and neopterin $(r=0.25, P=.10)$ were correlated with physical but not with mental fatigue. In cancer survivors, only IL-1-ra was related to both physical fatigue $(r=0.24, P=.10)$ and mental fatigue $(r$ $=0.35, P=.02$ ). CONCLUSIONS: In advanced cancer, inflammation seems to be associated with physical fatigue, but not to mental fatigue. In cancer survivors, there was no convincing evidence that inflammation plays a major role in fatigue. Cancer 2012;118:60056011. (C) 2012 American Cancer Society.
\end{abstract}

KEYWORDS: neoplasms, fatigue, mental fatigue, survivors, palliative care, C-reactive protein, interleukin-6, interleukin-8, interleukin-1 receptor antagonist protein, neopterin.

Fatigue is one of the most frequent and pervasive symptoms among cancer patients. Patients in all stages of the disease trajectory, from before diagnosis to years after treatment, experience fatigue, which has a greater negative impact on quality of life and daily functioning than any other cancer-related symptom. ${ }^{1}$ Patients describe their feelings of fatigue as physical, cognitive, and affective senses of tiredness, which indicates that fatigue is a multidimensional phenomenon. ${ }^{2}$

Although the exact pathogenesis of fatigue is still unknown, there is not much doubt about the multicausality of fatigue. ${ }^{3}$ Inflammation has been hypothesized to be one of the causes of cancer-related fatigue. ${ }^{3-6}$ In a review that pooled correlation coefficients between fatigue and cytokine serum levels of individual studies, a statistically significant positive association was found between fatigue and circulating levels of interleukin-6 (IL-6), interleukin-1 receptor antagonist (IL1-ra), and neopterin. ${ }^{5}$ These inflammatory markers may be produced by tumor cells or by inflammatory cells after their stimulation by antitumor treatment, tumor cells or psychological distress. ${ }^{6}$ They may provoke cancer-related fatigue by inducing anemia, disturbances in the hypothalamic-pituitary-adrenal axis or alterations in brain serotonin metabolism. ${ }^{4}$

Although a statistically significant association between inflammation and fatigue has been found in cancer patients at various stages of the disease trajectory, ${ }^{5}$ there are no studies that compared different groups of patients, ie, patients with advanced disease and cancer survivors, with respect to this association. Nevertheless, because tumor load, antitumor therapy, and distress are known to affect cytokine levels, ${ }^{6}$ it seems reasonable to suppose a different role of inflammation at various time points along the disease trajectory. Furthermore, most studies on cytokines and fatigue measured fatigue as a single symptom, whereas fatigue is known to have both physical and cognitive dimensions. These dimensions of fatigue

Corresponding author: Pleun J. de Raaf, MD, Department of Medical Oncology, Erasmus MC, Daniel den Hoed Cancer Center, PO Box 5201, 3008 AE Rotterdam, the Netherlands; Fax: +31-10-7041003; p.deraaf@erasmusmc.nl

${ }^{1}$ Department of Medical Oncology, Erasmus MC Daniel den Hoed Cancer Center, Rotterdam, the Netherlands; ${ }^{2}$ Laboratory of Clinical Tumor Immunology, Department of Medical Oncology, Erasmus MC Daniel den Hoed Cancer Center, Rotterdam, the Netherlands

The authors thank H. M. Kneefel for editing the graphs.

DOI: 10.1002/cncr.27613, Received: December 2, 2011; Revised: March 5, 2012; Accepted: March 27, 2012, Published online June 26, 2012 in Wiley Online Library (wileyonlinelibrary.com) 
might have different etiologies. For that reason, it is important to investigate whether inflammation is associated with all dimensions of fatigue or only with a single dimension.

In contrast to previous studies, we explored the association between fatigue and inflammation in patients with advanced cancer and in cancer survivors and for the physical and mental dimensions of fatigue separately. Based on the review of Schubert et al, we decided to measure plasma concentrations of IL-6, IL-1-ra, and neopterin, because these markers were found to be significantly correlated with fatigue. ${ }^{5}$ Furthermore, we measured C-reactive protein (CRP), because it is considered to be a surrogate marker of IL- 6 activity ${ }^{4}$ for which routine measurements are available in clinical practice. We also measured interleukin-8 (IL-8), because we previously found an increase in the concentration of this cytokine after infusion of taxanes, which are known to cause major fatigue (unpublished data). In these exploratory analyses, we aimed to identify inflammatory markers possibly involved in the pathogenesis of cancer-related fatigue, which should be studied in greater detail in subsequent studies. Therefore, we determined in both advanced cancer patients and cancer survivors: 1) which inflammatory markers are related to physical fatigue and mental fatigue, and 2) whether inflammatory markers that are associated with fatigue are related to each other.

\section{MATERIALS AND METHODS}

\section{Study Population}

Patients included in this study originated from an observational study on differences in fatigue experiences between advanced cancer patients and cancer survivors. ${ }^{7}$ Advanced cancer patients admitted to the palliative care unit or having an appointment at the outpatient clinic of the Erasmus MC Daniel den Hoed Cancer Centre, Rotterdam, the Netherlands, were invited to participate in the study. They were eligible when systemic anticancer therapy was no longer available and when they had not received any systemic anticancer therapy in the last 4 weeks.

For every advanced cancer patient, a cancer survivor matched for age, sex, and cancer diagnosis was recruited at the outpatient clinic. If feasible, they were also matched for treatment received for the primary tumor. These cancer survivors were eligible if their last treatment had been received 1 to 5 years ago and if there was no evidence of disease at the moment of inclusion. Patients still receiving adjuvant endocrine therapy for breast cancer were eligible for inclusion.
Patients for whom regular laboratory examinations were planned by their physician were asked permission for taking 1 extra blood sample for research on the relationship between fatigue and inflammatory markers. Here, data are published for the patients who both provided a blood sample and completed the fatigue questionnaire.

The Research Ethics Committee at the Erasmus MC granted ethical permission for this study, and all participants gave informed consent before data collection.

\section{Procedures}

All eligible participants were given oral and written information by the first author and were asked to give written consent. Venous blood samples, anticoagulated with ethylene diamine tetraacetic acid, were taken between $8 \mathrm{AM}$ and 12 noon in order to minimize the effects of diurnal fluctuations in cytokine levels. ${ }^{8}$ Plasma was harvested within 2 hours from collection, and stored in aliquots at $-80^{\circ} \mathrm{C}$ until analysis. Patients were requested to complete the fatigue questionnaire on the same day as the laboratory examination.

\section{Questionnaire}

Data on demographic characteristics and disease characteristics, including tumor type and anticancer therapy received, were collected from the electronic medical record.

The dimensions of fatigue were measured with the physical fatigue and mental fatigue subscales of the Multidimensional Fatigue Inventory (MFI). The subscale physical fatigue consists of 4 statements referring to the physical sensations related to subjective fatigue, and the subscale mental fatigue consists of 4 statements referring to cognitive symptoms (Table 1). Patients have to indicate to what extent each statement applied to them on a 5point Likert scale. Both subscale scores range from 4 to 20 , with higher score indicating more severe fatigue. Schwarz et al provided reference values for the MFI, determined in a sample of 2037 German representatives from the general population. ${ }^{10}$

\section{Measurement of Inflammatory Markers}

Plasma concentrations of CRP, IL-1-ra, and neopterin were measured by enzyme-linked immunosorbent assays using commercially available kits (CRP: Assay Pro, St Charles, Mo; IL-1-ra: R\&D Systems, Minneapolis, Minn; neopterin: IBL International, Hamburg, Germany) according to manufacturer instructions. IL- 6 and IL-8 plasma concentrations were determined by the Cytometric Bead Arrays (BD Bioscience, San Diego, Calif) according to the manufacturer instructions. Samples were tested in duplicate and related to the standard curves for 
Table 1. Items of the Subscales Physical Fatigue and Mental Fatigue of the Multidimensional Fatigue Inventory ${ }^{9}$

\section{Physical Fatigue}

1. Physically I feel only able to do a little

2. Physically I can take on a lot

3. Physically I feel I am in a bad condition

4. Physically I feel I am in an excellent condition

\section{Mental Fatigue}

1. When I am doing something, I can keep my thoughts on it

2. I can concentrate well

3. It takes a lot of effort to concentrate on things

4. My thoughts easily wander each assay. The lowest detection limit per assay was 0.25 $\mathrm{ng} / \mathrm{mL}$ for CRP; $1.35 \mathrm{nmol} / \mathrm{L}$ for neopterin; $15.6 \mathrm{pg} / \mathrm{mL}$ for IL-1-ra; $5.0 \mathrm{pg} / \mathrm{mL}$ for IL-6; and $2.5 \mathrm{pg} / \mathrm{mL}$ for IL-8. If the level of an inflammatory marker was undetectable, the detection limit for that cytokine was imputed in the data set.

\section{Data Analyses}

Because this study is a secondary analysis of a previous study, we did not perform a power analysis. Because of the explorative character of this study, $P$ values were not corrected for multiple testing and associations with $P<0.10$ are considered to be important to investigate in further research. Differences in patients' and disease characteristics between advanced cancer patients and cancer survivors were assessed with the chi-square test. Differences between advanced cancer patients and cancer survivors in the intensity of fatigue and the levels of the inflammatory markers were determined with the Mann-Whitney U test. For both advanced cancer patients and cancer survivors, Spearman correlation coefficients were calculated to explore the relationships between fatigue measurements and concentrations of inflammatory markers.

\section{RESULTS}

We included a total of 63 advanced cancer patients and 63 cancer survivors in the original study between October 2008 and October 2010. Eighteen advanced cancer patients and 16 cancer survivors did not provide a blood sample, because their physician had not planned a regular laboratory examination. In this study, we present the data of the 45 advanced cancer patients and the 47 cancer survivors who both completed the questionnaire and provided a blood sample. In this analysis, 12 advanced cancer patients and 14 cancer survivors were unmatched because their match did not provide a blood sample, 22 pairs of patients were matched for age, sex, and diagnosis, and 11 pairs of patients were matched for age, sex, diagnosis, and treatment of primary tumor. Patient characteristics and fatigue scores are presented in Table 2. There were no significant differences between the groups in age, sex, or tumor diagnosis. Advanced cancer patients had a signifi- cantly lower physical performance than cancer survivors $(P<.01)$. Advanced cancer patients had undergone primary surgery less frequently than cancer survivors $(P<$ .01 ), because $18 \%$ of advanced cancer patients already had advanced disease at the time of diagnosis. Advanced cancer patients had been treated with chemotherapy and radiotherapy more frequently than cancer survivors $(P<$ $.05)$. Compared to cancer survivors, advanced cancer patients had higher levels of physical fatigue (median, 16 vs $9, P<.001$ ) and mental fatigue (median 11 vs 6 , $P=.01)$.

Levels of inflammatory markers for advanced cancer patients and cancer survivors are shown in Figure 1. Levels of all inflammatory markers were higher in advanced cancer patients than in cancer survivors $(P<.01)$. A total of $93 \%$ of advanced cancer patients and $21 \%$ of cancer survivors had at least 1 marker above the upper limit of the normal range $(P<.001)$. Seventy-three percent of advanced cancer patients and $6 \%$ of cancer survivors had at least 1 marker at 2 times higher than the upper limit of the normal range $(P<.001)$.

The relationships between fatigue measurements and levels of inflammatory markers for both advanced cancer patients and cancer survivors are shown in Figure 2 . In the advanced cancer patients, physical fatigue was positively correlated with the levels of CRP $(\mathrm{r}=0.49, P=$ $.001)$, IL-6 ( $\mathrm{r}=0.43, P=.003)$, IL-1-ra $(\mathrm{r}=0.32, P=$ $.03)$, and, to a lesser extent, neopterin $(\mathrm{r}=0.25, P=.10)$. No inflammatory markers were related to mental fatigue in the advanced cancer patients. In the cancer survivors, IL-1-ra was related to both physical fatigue ( $\mathrm{r}=0.24, P=$ $.10)$ and mental fatigue $(\mathrm{r}=0.35, P=.02)$. In this group, no associations were found for any other soluble factors assessed with either physical or mental fatigue.

All inflammatory markers that were associated with physical fatigue in the advanced cancer patients were positively correlated with each other as detailed in Table 3.

\section{DISCUSSION}

In this study, we explored whether fatigue dimensions were associated with inflammatory markers in different 
Table 2. Patient Characteristics

\begin{tabular}{|c|c|c|}
\hline \multirow[t]{2}{*}{ Characteristic } & $\begin{array}{l}\text { Advanced } \\
\text { Cancer } \\
\text { Patients }\end{array}$ & $\begin{array}{l}\text { Cancer } \\
\text { Survivors }\end{array}$ \\
\hline & $\begin{array}{l}(n=45) \\
N(\%)\end{array}$ & $\begin{array}{l}(\mathrm{n}=47) \\
\mathrm{N}(\%)\end{array}$ \\
\hline Age, y (mean, range) & $58(22-81)$ & $57(36-77)$ \\
\hline \multicolumn{3}{|l|}{ Sex } \\
\hline Male & $18(40 \%)$ & $19(40 \%)$ \\
\hline Female & $27(60 \%)$ & $28(60 \%)$ \\
\hline \multicolumn{3}{|l|}{ WHO performance ${ }^{a}$} \\
\hline 0 & $1(2 \%)$ & $29(62 \%)$ \\
\hline 1 & $16(36 \%)$ & $17(36 \%)$ \\
\hline 2 & 17 (39\%) & $1(2 \%)$ \\
\hline 3 & $8(18 \%)$ & \\
\hline 4 & $2(5 \%)$ & \\
\hline \multicolumn{3}{|l|}{ Cancer diagnosis } \\
\hline Breast & $15(33 \%)$ & $15(32 \%)$ \\
\hline Gastrointestinal & $11(24 \%)$ & $14(30 \%)$ \\
\hline Urogenital & 9 (20\%) & $8(17 \%)$ \\
\hline Other & $10(22 \%)$ & $10(21 \%)$ \\
\hline \multicolumn{3}{|l|}{ Treatment received } \\
\hline \multicolumn{3}{|l|}{ Surgery } \\
\hline Total & $33(73 \%)$ & $46(98 \%)^{a}$ \\
\hline Curative & $32(71 \%)$ & $46(98 \%)^{a}$ \\
\hline Palliative & $11(24 \%)$ & \\
\hline \multicolumn{3}{|l|}{ Chemotherapy } \\
\hline Total & 37 (82\%) & $30(64 \%)^{b}$ \\
\hline Curative & $17(38 \%)$ & $30(64 \%)^{b}$ \\
\hline Palliative & 31 (69\%) & \\
\hline \multicolumn{3}{|l|}{ Radiotherapy } \\
\hline Total & $33(73 \%)$ & $24(51 \%)^{\mathrm{b}}$ \\
\hline Curative & $19(42 \%)$ & 24 (51\%) \\
\hline Palliative & 22 (49\%) & \\
\hline \multicolumn{3}{|l|}{ Hormonal therapy } \\
\hline Total & $13(29 \%)$ & $14(30 \%)$ \\
\hline Curative & $6(13 \%)$ & $14(30 \%)$ \\
\hline Palliative & $12(27 \%)$ & \\
\hline $\begin{array}{l}\text { Months since last treatment } \\
\quad(\text { median, IQR) }\end{array}$ & $2.5(1.0-7.0)$ & $28.0(20.0-47.0)$ \\
\hline \multicolumn{3}{|c|}{ Fatigue scores (median, IQR) } \\
\hline Physical fatigue & $16(13-19)$ & $9(5-12)^{b}$ \\
\hline Mental fatigue & $11(6-15)$ & $6(4-12)^{b}$ \\
\hline
\end{tabular}

IQR indicates interquartile range; WHO, World Health Organization.

a $P<0.01$

${ }^{\mathrm{b}} P<0.05$

${ }^{\mathrm{C}}$ Surgery, chemotherapy, or radiotherapy.

groups of cancer patients. In the advanced cancer patients, CRP, IL-6, IL-1-ra, and neopterin were significantly associated with physical fatigue but not with mental fatigue. These findings suggest that physical fatigue and mental fatigue have different underlying pathogeneses in these patients. On the contrary, in the cancer survivors, both physical fatigue and mental fatigue were associated with IL-1-ra only, which indicates that the pathogenesis of physical and mental fatigue might differ between advanced cancer patients and cancer survivors.

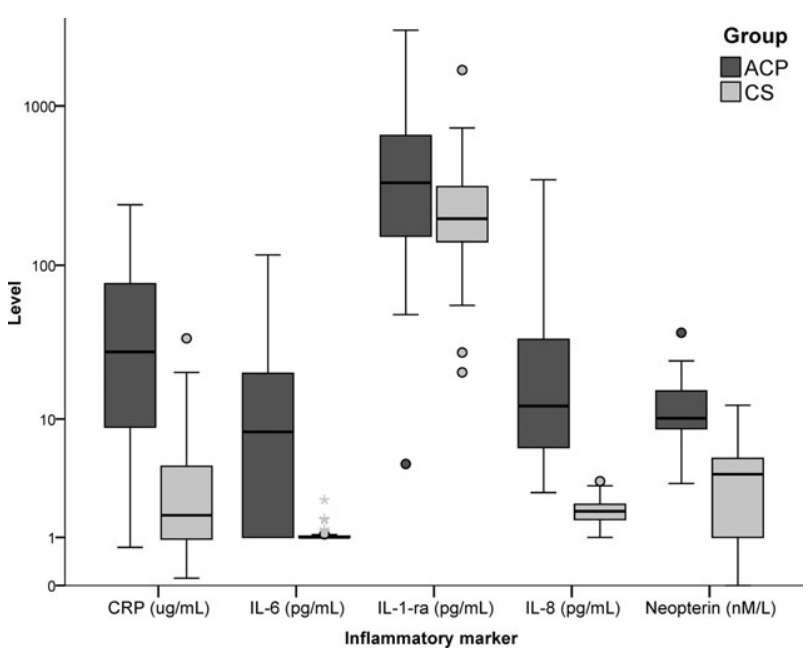

Figure 1. Box plot of plasma concentrations of C-reactive protein (CRP), interleukin-6 (IL-6), interleukin-1 receptor antagonist (IL-1-ra), interleukin-8 (IL-8), and neopterin in advanced cancer patients (ACP) and cancer survivors (CS). Horizontal line indicates median; box is interquartile range; whiskers indicate range (without outliers); dots indicate outliers ( $>1.5$ times interquartile range above 75 th or below 25 th percentile).

Four other studies have previously investigated the relation between at least 1 of these inflammatory markers and fatigue in advanced cancer patients. ${ }^{11-14}$ The only study that measured fatigue multidimensionally in terminally ill advanced cancer patients also found the IL- 6 concentrations to be related to physical fatigue and not to mental fatigue. ${ }^{11}$ The 3 other studies measured fatigue as 1 symptom (unidimensionally), and their results were in contrast with our findings. ${ }^{12-14}$ Two of these studies investigated the relation between IL- 6 and fatigue in advanced cancer patients and could not find a significant relation. ${ }^{13,14}$ However, these patients had a better performance than our patients $\left(93 \%{ }^{14}\right.$ and $75 \%{ }^{13}$ Eastern Cooperative Oncology Group performance status [ECOG PS] $\leq 1$ vs $38 \%$ ECOG PS $\leq 1$ in our study). Therefore, our patients were more vulnerable and might have experienced more fatigue. The third study investigated the relation between CRP and symptom clusters in patients with advanced cancer of the lung or pancreas. Fatigued patients had similar CRP concentrations as nonfatigued patients. Furthermore, in this study, hardly any patient had a CRP concentration above the upper limit of the normal range $(10 \mu \mathrm{g} / \mathrm{mL}) .{ }^{12}$ No studies assessing the relationship between fatigue and IL-1-ra, IL-8, or neopterin in advanced cancer patients have been published thus far.

In the advanced cancer patients, all inflammatory markers that were associated with physical fatigue were 


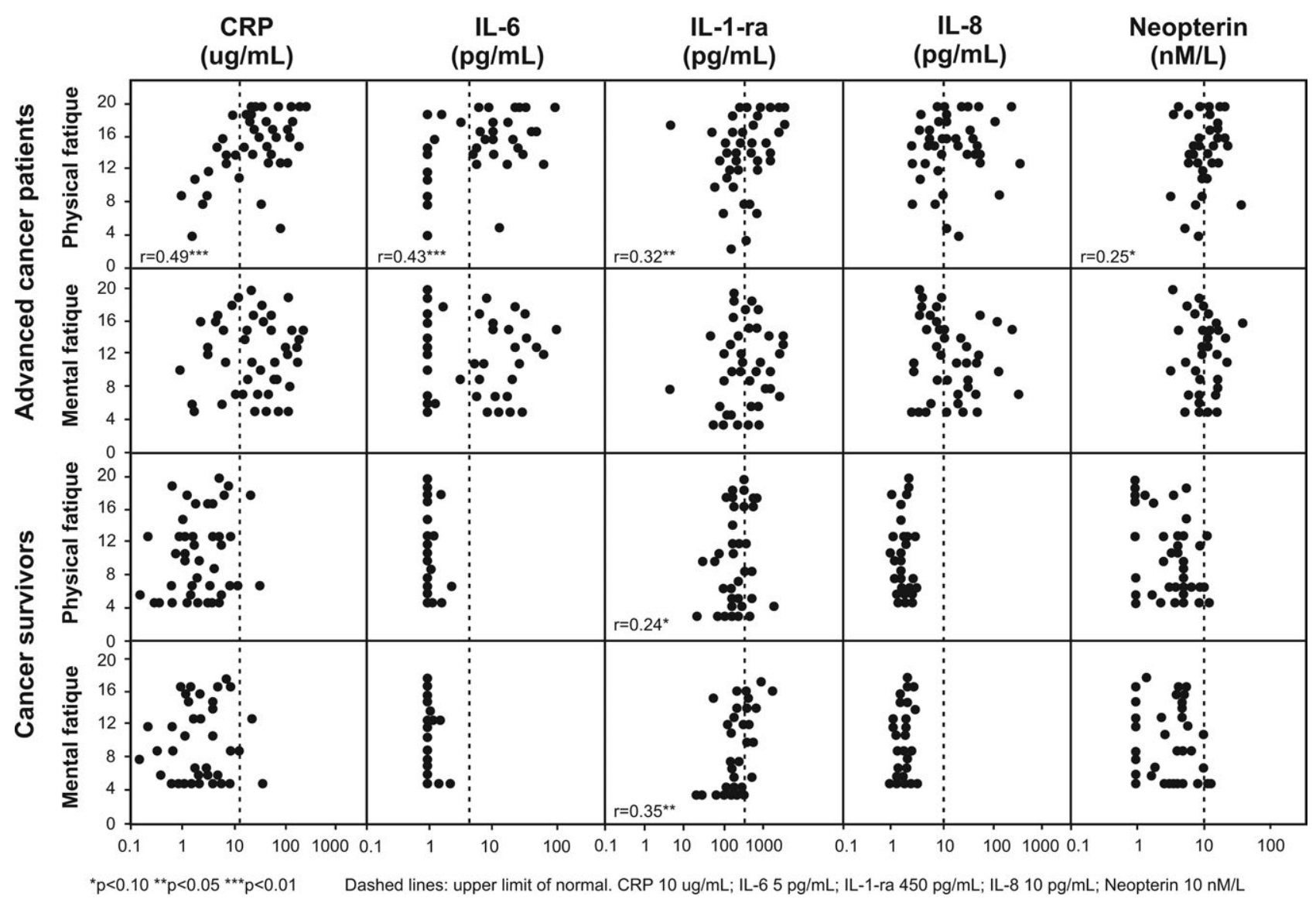

Figure 2. Scatter plots of the fatigue scores and plasma concentrations of inflammatory markers, including C-reactive protein (CRP), interleukin-6 (IL-6), interleukin-1 receptor antagonist (IL-1-ra), interleukin-8 (IL-8), and neopterin, of individual patients. The Spearman correlation coefficient is presented for significant correlations.

Table 3. Spearman Correlations Between Inflammatory Markers Significantly Associated With Physical Fatigue in Advanced Cancer Patients

$\begin{array}{llll} & \text { IL-6 } & \text { IL-1-ra } & \text { Neopterin } \\ \text { C-Reactive protein } & 0.82^{\mathrm{a}} & 0.50^{\mathrm{a}} & 0.35^{\mathrm{b}} \\ \text { IL-6 } & - & 0.58^{\mathrm{a}} & 0.36^{\mathrm{b}} \\ \text { IL-1-ra } & & - & 0.27^{\mathrm{c}} \\ \text { Neopterin } & & & -\end{array}$

${ }^{\mathrm{a}} P<0.01$.

${ }^{\mathrm{b}} P<0.05$.

${ }^{\mathrm{c}} P<0.1$.

positively intercorrelated. CRP is an acute-phase protein produced by hepatocytes after stimulation by IL- 6 , which is reflected by a strong correlation between CRP and IL-6 in our study. ${ }^{15}$ IL-6 production is stimulated by IL- $1 \beta .{ }^{16}$ Both IL- $1 \beta$ and IL- 6 stimulate the production of IL-1-ra, an anti-inflammatory cytokine that antagonizes IL-1 activity. ${ }^{17}$ Therefore, of the markers that were correlated with fatigue in our study, IL-6 in particular might play an important role in the fatigue-inducing cytokine cascade.
Subsequently, the elevated levels of both CRP and IL-1-ra in this study could be caused by increased IL- 6 activity. Interestingly, in the treatment of IL-6-mediated Castleman's disease, both blockade of IL- 6 production by IL-1ra administration ${ }^{18}$ and blockade of IL- 6 activity by administration of anti-IL-6 antibodies ${ }^{19}$ were highly effective in decreasing disease activity and in alleviating fatigue. Furthermore, there are some data from phase 1 and phase 2 clinical trials which suggest that anti-IL- 6 antibody treatment in patients with advanced lung cancer might alleviate fatigue. ${ }^{20}$

The association between inflammatory markers and fatigue in cancer survivors was investigated previously in 6 studies. ${ }^{21-26}$ In the only study that measured fatigue multidimensionally, IL-1-ra was associated with physical fatigue but not with mental fatigue, whereas we found an association with both physical and mental fatigue. Furthermore, this study found a significant correlation between CRP and physical fatigue, whereas we did not. ${ }^{25}$ The 5 other studies in cancer survivors measured fatigue 
only unidimensionally. ${ }^{21-24,26}$ In a study on breast cancer survivors, CRP was associated with fatigue, in contrast to our results. ${ }^{26}$ Similar to our results, there was no relation between IL- 6 and fatigue in other studies on cancer survivors. ${ }^{22-26}$ The results of the studies on IL-1-ra and fatigue and on neopterin and fatigue in cancer survivors were contradictory. Some studies found an association between fatigue and IL-1-ra ${ }^{21,22}$ or neopterin, ${ }^{23,25,26}$ whereas other studies did not find an association between fatigue and IL-1-ra ${ }^{23,26}$ or neopterin. ${ }^{21}$ The association between IL-8 and fatigue has never been studied before in cancer survivors.

The clinical meaning of the solitary association of IL-1-ra and fatigue in the cancer survivors remains unclear. Because this relation is congruent with the literature, ${ }^{21,22,25}$ it seems unlikely that the statistically significant correlation we found is caused by chance, because of multiple testing. On the other hand, if inflammation is one of the important causes of fatigue in the cancer survivors, we would have expected more inflammatory markers to be related to fatigue, because they are not independent factors, but part of complex, collaborating pathways.

There are several limitations to this study, hindering the interpretation of the results. Because of the cross-sectional study design, we were not able to prove causality between inflammation and physical fatigue. Longitudinal measurements of both fatigue severity and concentrations of inflammatory markers are needed to confirm the causal relationship between them. Furthermore, despite our matching advanced cancer patients and cancer survivors to minimize intergroup heterogeneity, there is a great intragroup variability in age, diagnosis, and intensity of treatment. Finally, in this explorative study, we did not control for other variables that are known to influence fatigue, such as depression. Depression has been reported to be correlated with both physical and mental fatigue in patients with advanced cancer ${ }^{27}$ as well as cancer survivors. ${ }^{28}$ Also, behavioral symptoms such as depression have been hypothesized to be related to cytokine release. ${ }^{29}$ However, a recent study in breast cancer patients who completed curative chemotherapy recently failed to find a correlation between depression and inflammatory activity; whereas unidimensionally measured fatigue was correlated with both inflammation and depression. ${ }^{30}$ These results further substantiate the multicausality of cancerrelated fatigue and the need for large, prospective studies to unravel the etiologic mechanisms.

Nevertheless, the novelty of this study lies in the simultaneous measurement of the relation between inflammation and fatigue dimensions in different groups of cancer patients. We showed that inflammation seems to be associated with physical fatigue, but not to mental fatigue in the advanced cancer patients, whereas there were no strong indications that inflammation plays a major role in cancer survivors' fatigue. In future research, longitudinal studies controlling for important confounders are needed to prove the causal relationship between inflammation and fatigue. Furthermore, if it should be proven that cytokines cause fatigue, it has to be investigated which pathogenetic pathways lead from elevated concentrations of inflammatory markers in plasma to subjective complaints of fatigue. Hopefully, the identification of the important inducers of cancer-related fatigue will finally lead to novel targets of treatment, for example by blockade of IL-6 production or IL-6 activity.

\section{FUNDING SOURCES}

Financial support for this study was provided by Stichting Cornelis Vrolijk Development Fund (nonprofit, nongovernmental organization).

\section{CONFLICT OF INTEREST DISCLOSURE}

The authors made no disclosure.

\section{REFERENCES}

1. Hofman M, Ryan JL, Figueroa-Moseley CD, Jean-Pierre P, Morrow GR. Cancer-related fatigue: the scale of the problem. Oncologist. 2007;12(suppl 1):4-10.

2. Glaus A, Crow R, Hammond S. A qualitative study to explore the concept of fatigue/tiredness in cancer patients and in healthy individuals. Support Care Cancer. 1996;4:82-96.

3. Ryan JL, Carroll JK, Ryan EP, Mustian KM, Fiscella K, Morrow GR. Mechanisms of cancer-related fatigue. Oncologist. 2007;12(suppl 1): 22-34.

4. Jager A, Sleijfer S, van der Rijt CC. The pathogenesis of cancer related fatigue: could increased activity of pro-inflammatory cytokines be the common denominator? Eur J Cancer. 2008;44:175181.

5. Schubert C, Hong S, Natarajan L, Mills PJ, Dimsdale JE. The association between fatigue and inflammatory marker levels in cancer patients: a quantitative review. Brain Behav Immun. 2007;21:413427.

6. Seruga B, Zhang H, Bernstein LJ, Tannock IF. Cytokines and their relationship to the symptoms and outcome of cancer. Nat Rev Cancer. 2008;8:887-899.

7. de Raaf PJ, de Klerk C, Timman R, Hinz A, van der Rijt CCD. Differences in fatigue experiences between advanced cancer patients, cancer survivors and the general population. J Pain Symptom Manage. 2012; doi: 10.1016/j.jpainsymman.2011.12.279.

8. Lange T, Dimitrov S, Born J. Effects of sleep and circadian rhythm on the human immune system. Ann N Y Acad Sci. 2010;1193:4859.

9. Smets EM, Garssen B, Bonke B, De Haes JC. The Multidimensional Fatigue Inventory (MFI) psychometric qualities of an instrument to assess fatigue. J Psychosom Res. 1995;39:315-325.

10. Schwarz R, Krauss O, Hinz A. Fatigue in the general population. Onkologie. 2003;26:140-144.

11. Inagaki $M$, Isono M, Okuyama T, et al. Plasma interleukin-6 and fatigue in terminally ill cancer patients. J Pain Symptom Manage. 2008;35:153-161. 
12. Laird BJ, Scott AC, Colvin LA, et al. Pain, depression, and fatigue as a symptom cluster in advanced cancer. J Pain Symptom Manage. 2011;42:1-11.

13. Mantovani G, Maccio A, Madeddu C, et al. A phase 2 study with antioxidants, both in the diet and supplemented, pharmaconutritional support, progestagen, and anti-cyclooxygenase- 2 showing efficacy and safety in patients with cancer-related anorexia/cachexia and oxidative stress. Cancer Epidemiol Biomarkers Prev. 2006;15:1030-1034.

14. Rich T, Innominato PF, Boerner J, et al. Elevated serum cytokines correlated with altered behavior, serum cortisol rhythm, and dampened 24-hour rest-activity patterns in patients with metastatic colorectal cancer. Clin Cancer Res. 2005;11:1757-1764.

15. Gabay C, Kushner I. Acute-phase proteins and other systemic responses to inflammation. $N$ Engl J Med. 1999;340:448-454.

16. Dinarello CA. Biologic basis for interleukin-1 in disease. Blood. 1996;87:2095-2147.

17. Gabay C, Smith MF, Eidlen D, Arend WP. Interleukin 1 receptor antagonist (IL-1Ra) is an acute-phase protein. J Clin Invest. 1997; 99:2930-2940.

18. El-Osta H, Janku F, Kurzrock R. Successful treatment of Castleman's disease with interleukin-1 receptor antagonist (Anakinra). Mol Cancer Ther. 2010;9:1485-1488.

19. Nishimoto N, Kanakura Y, Aozasa K, et al. Humanized anti-interleukin-6 receptor antibody treatment of multicentric Castleman disease. Blood. 2005;106:2627-2632.

20. Bayliss TJ, Smith JT, Schuster M, Dragnev KH, Rigas JR. A humanized anti-IL-6 antibody (ALD518) in non-small cell lung cancer. Expert Opin Biol Ther. 2011;11:1663-1668.

21. Bower JE, Ganz PA, Aziz N, Fahey JL. Fatigue and proinflammatory cytokine activity in breast cancer survivors. Psychosom Med. 2002;64:604-611.
22. Collado-Hidalgo A, Bower JE, Ganz PA, Cole SW, Irwin MR Inflammatory biomarkers for persistent fatigue in breast cancer survivors. Clin Cancer Res. 2006;12:2759-2766.

23. Dimeo F, Schmittel A, Fietz T, et al. Physical performance, depression, immune status and fatigue in patients with hematological malignancies after treatment. Ann Oncol. 2004;15:1237-1242.

24. Knobel H, Loge JH, Nordoy T, et al. High level of fatigue in lymphoma patients treated with high dose therapy. J Pain Symptom Manage. 2000;19:446-456.

25. Orre IJ, Murison R, Dahl AA, Ueland T, Aukrust P, Fossa SD. Levels of circulating interleukin-1 receptor antagonist and C-reactive protein in long-term survivors of testicular cancer with chronic cancer-related fatigue. Brain Behav Immun. 2009;23:868-874.

26. Orre IJ, Reinertsen KV, Aukrust P, et al. Higher levels of fatigue are associated with higher CRP levels in disease-free breast cancer survivors. J Psychosom Res. 2011;71:136-141.

27. Munch TN, Strömgren AS, Pedersen L, Petersen MA, Hoermann L, Groenvold M. Multidimensional measurement of fatigue in advanced cancer patients in palliative care: an application of the multidimensional fatigue inventory. J Pain Symptom Manage. 2006; 31:533-541.

28. Kuhnt S, Ernst J, Singer S, et al. Fatigue in cancer survivors-prevalence and correlates. Onkologie. 2009;32:312-317.

29. Miller AH, Ancoli-Israel S, Bower JE, Capuron L, Irwin MR. Neuroendocrine-immune mechanisms of behavioral comorbidities in patients with cancer. J Clin Oncol. 2008;26:971-982.

30. Bower JE, Ganz PA, Irwin MR, Kwan L, Breen EC, Cole $\mathrm{SW}$. Inflammation and behavioral symptoms after breast cancer treatment: do fatigue, depression, and sleep disturbance share a common underlying mechanism? J Clin Oncol. 2011;29:35173522 . 